\title{
Synaptic Protein Dynamics in Hibernation
}

\author{
Christina G. von der Ohe, ${ }^{1}$ Craig C. Garner, ${ }^{3}$ Corinna Darian-Smith, ${ }^{2}$ and H. Craig Heller ${ }^{1}$ \\ Departments of ${ }^{1}$ Biological Sciences, ${ }^{2}$ Comparative Medicine, and ${ }^{3}$ Psychiatry and Behavioral Sciences, Stanford University, Stanford, California 94305
}

\begin{abstract}
Neurons in hibernating mammals exhibit a dramatic form of plasticity during torpor, with dendritic arbors retracting as body temperature cools and then regrowing rapidly as body temperature rises. In this study, we used immunohistochemical imaging and Western blotting of several presynaptic and postsynaptic proteins to determine the synaptic changes that accompany torpor and to investigate the mechanisms behind these changes. We show torpor-related alterations in synaptic protein localization that occur rapidly and uniformly across several brain regions in a temperature-dependent manner. Entry into torpor is associated with a $50-65 \%$ loss of synapses, as indicated by changes in the extent of colocalization of presynaptic and postsynaptic markers. We also show that the loss of synaptic protein clustering occurring during entry into torpor is not attributable to protein loss. These findings suggest that torpor-related changes in synapses stem from dissociation of proteins from the cytoskeletal active zone and postsynaptic density, creating a reservoir of proteins that can be quickly mobilized for rapid rebuilding of dendritic spines and synapses during the return to euthermia. A mechanism of neural plasticity based on protein dissociation rather than protein breakdown could explain the hibernator's capacity for large, rapid, and repeated microstructural changes, providing a fascinating contrast to neuropathologies that are dominated by protein breakdown and cell death.
\end{abstract}

Key words: synapse; protein; hibernation; torpor; temperature; plasticity

\section{Introduction}

Hibernating mammals survive extreme, repeated drops in body temperature that can reach near-freezing levels (Strumwasser, 1959; Wang, 1973; Barnes, 1989). During deep torpor, there is a virtual cessation of neural activity in the brain (Strumwasser, 1959; Shtark, 1970; Walker et al., 1977). These dramatic changes in tissue temperature and neural activity are paralleled by changes in neuronal microstructure, in which alterations in cell body area, dendritic arbor complexity, and spine density are observed (Popov et al., 1992; von der Ohe et al., 2006). Torpor-related neural changes have also been demonstrated at the molecular level, as shown by immunohistochemical analysis of hippocampal polysialylated neural cell adhesion molecule, synaptophysin, and microtubule-associated protein 2 (MAP2) (Arendt et al., 2003). These studies have shown that the brain of the hibernating mammal is a model of rapid and reversible neural plasticity, with rates of change that are among the most dramatic found in nature, particularly among adult mammals. The hibernator promises to be an informative model of neural restructuring in the adult brain, with the potential to advance our understanding of processes including hypothermia, learning and memory, and neurodegeneration. To test the potential of the hibernator to

Received July 6, 2006; revised Nov. 16, 2006; accepted Nov. 21, 2006.

This work was supported by grants from the Society for Integrative and Comparative Biology and National Institutes of Health Grant R21 NS051128-01A1. We are grateful to F. Fernandez, V. Torres, and M. Grolle for helping with the Western blots and immunohistochemistry; M. Lopez for performing the telemeter implant surgeries; $\mathrm{N}$. Ruby, S. Ortiz, C. Specht, and C. Brüderle for technical assistance; R. Sapolsky, R. Fernald, P. Bourgin, and P. Gratzinger for critical reading of this manuscript; and many of our colleagues for their suggestions and discussion.

Correspondence should be addressed to Christina G. von der Ohe, Department of Biological Sciences, Stanford University, Stanford, CA 94305-5020. E-mail: vonderohe@stanford.edu.

D0I:10.1523/JNEUROSCI.4385-06.2007

Copyright $\odot 2007$ Society for Neuroscience $\quad$ 0270-6474/07/270084-09\$15.00/0 shed light on these topics, we studied the effects of temperature on torpor-related synaptic plasticity, the extent of loss of synapses in torpor, and the mechanism of synaptic protein loss.

In the present study, we investigated the dynamics of torporrelated changes in synaptic protein localization and composition by analyzing several presynaptic and postsynaptic proteins over the course of torpor. To confirm that synaptic protein dynamics reflect the changes we reported for dendritic arbors and spines during hibernation (von der Ohe et al., 2006), we assessed whether synaptic changes are occurring similarly across various brain regions and whether they occur in a temperaturedependent manner. To determine the extent of synaptic restructuring that takes place during arousal, we investigated how changes in synaptic protein immunoreactivity relate to the number of synapses, as defined by rates of colocalization between presynaptic and postsynaptic markers. We also assessed whether the loss of synaptic protein clustering demonstrated by immunohistochemistry is attributable to loss of protein or to dissociation of proteins from discrete puncta. We show that neural plasticity in the hibernator is a function of temperature and appears to result from rapid dissociation of proteins from synapses. The presence of this pool of synaptic proteins during torpor may account for the rapid rebuilding of dendritic spines and synapses that occurs during the return to euthermia.

\section{Materials and Methods}

Animals. All procedures were approved by the Institutional Animal Care and Use Committee of Stanford University. Golden-mantled ground squirrels (Spermophilus lateralis) were trapped in the Sierra Nevada, California (permit obtained from California Department of Fish and Game). To precisely monitor torpor bouts, animals were implanted with abdominal temperature telemeters (Mini-Mitter, Bend, OR). These $20 \mathrm{~mm}$ devices did not interfere with animal behavior or health. In the winter, the 
ambient temperature was decreased to $5^{\circ} \mathrm{C}$ and the lighting was changed to constant, dim red light. These conditions permitted hibernation in all of the animals. Subsequently, the ambient temperature was raised to $15^{\circ} \mathrm{C}$ for the month of February, after which it was lowered again to $5^{\circ} \mathrm{C}$. The body temperature of each animal was monitored remotely to assess torpor bout length. All animals completed a minimum of two full bouts of torpor before they were killed.

Experimental design. Seven experimental groups of six animals were used for the immunohistochemical study. Five of these groups hibernated at $5^{\circ} \mathrm{C}$, and two of these groups hibernated at $15^{\circ} \mathrm{C}$ before being killed. At $5^{\circ} \mathrm{C}$, the groups were 1,3 , and $6 \mathrm{~d}$ after torpor entrance and 2 and $12 \mathrm{~h}$ after arousal induction. These time points correspond to the start and end of both torpor and interbout euthermia at this temperature. Entry into torpor was defined as a drop of body temperature below $34^{\circ} \mathrm{C}$. Arousal from torpor was initiated by 2 min of gentle handling, a technique that induced complete arousal to above $34^{\circ} \mathrm{C}$ in $\sim 2 \mathrm{~h}$. For the two euthermic groups of animals at this temperature, arousal was induced at $6 \mathrm{~d}$ of torpor to ensure that all animals in the euthermic groups spent the same amount of time in torpor during that cycle. The two groups of animals hibernating at $15^{\circ} \mathrm{C}$ were $3 \mathrm{~d}$ after torpor entrance and $12 \mathrm{~h}$ after arousal induction. For the $15^{\circ} \mathrm{C}$ euthermic group, arousal was induced at $3 \mathrm{~d}$ of torpor, which is the approximate torpor bout length at that temperature.

Immunohistochemistry. Brain tissue was collected at specific times in the torpor bout ( $n=6$ animals per group). Animals were removed from their cages, deeply anesthetized with halothane for $1 \mathrm{~min}$, and killed by perfusion through the heart with heparinized PBS, followed by $4 \%$ paraformaldehyde in phosphate buffer, $\mathrm{pH}$ 7.4. Brains were removed and postfixed for $6-7 \mathrm{~h}$ in the same fixative and then transferred to PBS. Sequential $100 \mu \mathrm{m}$ coronal slices were cut on a vibratome in cold PBS. Slices were taken from two regions of the brain: (1) the anterior thalamus, including the reticular nucleus and somatosensory cortex, and (2) the posterior thalamus, including the ventral posterior thalamus and hippocampus. Slices were cut from the same regions across animals. Tissue was stored in $4 \%$ paraformaldehyde in PBS at $4^{\circ} \mathrm{C}$ until immunohistochemical processing, conducted $\sim 1$ week later. Free-floating slices were rinsed four times for 5 min with PBS and $0.1 \%$ Triton X-100, blocked with $3 \%$ goat serum, incubated overnight at $4^{\circ} \mathrm{C}$ in primary antibody, and incubated for an additional $1 \mathrm{~h}$ with secondary antibody. Slices were mounted onto pretreated slides with Vectashield (Vector Laboratories, Burlingame, CA). Slides were stored flat at $4^{\circ} \mathrm{C}$ until imaging, $\sim 1-2$ weeks later. Double labeling was performed for Piccolo and postsynaptic density 95 (PSD95) (see below, Antibodies used).

Slices were imaged on a Yokayama spinning disc confocal microscope and analyzed using Openlab 3.5.1 software (Scientific Software, Pleasanton, CA). Images were taken at $63 \times$, and all exposure settings were kept constant during image acquisition. Three $625 \mu \mathrm{m}^{2}$ squares were drawn on each image. Three parameters in each square were measured: (1) fluorescence intensity, which estimates the number of synaptic protein molecules expressed in the square, (2) number of discrete puncta, and (3) the proportion of the square that was covered by protein clusters. The number of puncta and area covered by protein clusters were measured after deletion of background levels of fluorescence via a sliding intensity bar, so that only discrete puncta remained. Fluorescence intensity was measured without background subtraction to determine whether total fluorescence in the slices changed with condition. For double-labeled images, 50 randomly selected puncta were analyzed for overlap. In all analyses, the investigator was blind to the experimental group of the animal. We analyzed slices from both euthermic and hibernating animals in which the primary antibody was not applied and found no difference in native fluorescence between these groups.

Antibodies used. Unless otherwise specified, the following primary and secondary antibodies were used in the present investigation: MAP2 (rabbit, 1:2000; Craig Garner, Stanford University, Stanford, CA); Piccolo (rabbit, 1:500; Craig Garner); PSD95 (mouse 05-494, 1:100; Millipore, Bedford, MA); synaptophysin (mouse VAM-SV011E, 1:100; Stressgen Biotechnologies, Victoria, British Columbia, Canada); and goat antirabbit Alexa 568, goat anti-mouse Alexa 568, and goat anti-mouse Alexa 488 (Invitrogen, Carlsbad, CA). Controls were tested to ensure minimal cross-reactivity. The rationale for the choice of proteins investigated is presented in Results.

Quantitative Western blot. Two cohorts of animals hibernating at $5^{\circ} \mathrm{C}$ were killed by rapid decapitation $12 \mathrm{~h}$ after arousal or $1 \mathrm{~d}$ after torpor entrance ( $n=3$ animals per group). Brains were immediately removed, flash frozen in liquid nitrogen, and stored at $-80^{\circ} \mathrm{C}$. Subsequently, a section of the brain containing somatosensory cortex, hippocampus, and thalamus was dissected and homogenized in buffer [ $40 \mathrm{~mm}$ Tris, $\mathrm{pH} 7.5$, $0.3 \mathrm{M}$ sucrose, $1.6 \mathrm{mg} / \mathrm{ml}$ protease inhibitor cocktail (Roche Applied Science, Indianapolis, IN), $5 \mathrm{~mm}$ EDTA, and phosphatase inhibitors: 1 mм NaF, 1 mm Na-molybdate, $1 \mathrm{~mm}$ Na-tartrate, 1 mm Na $\mathrm{VO}_{4}, 100 \mathrm{~nm}$ fenvalerate, and $250 \mathrm{~nm}$ okadaic acid (Sigma, St. Louis, MO) (ratio of tissue to buffer, 1:20)]. After centrifugation $\left(1000 \times g, 15 \mathrm{~min}, 4^{\circ} \mathrm{C}\right)$, the supernatant was collected, and several aliquots were kept for analysis of whole-brain protein content. The remainder of the supernatant, in combination with additional supernatant from a pellet wash, was centrifuged at 17,300 $\times g$ for $20 \mathrm{~min}$, using a fixed 50.2 Ti Beckman Coulter (Fullerton, $\mathrm{CA}$ ) rotor $\left(4^{\circ} \mathrm{C}\right)$. The resulting pellet was gently resuspended in $6 \mathrm{ml}$ of homogenizing buffer, loaded onto discontinuous sucrose gradients stacked from top to bottom with $7 \mathrm{ml}$ of $0.8,1.0$, and $1.2 \mathrm{M}$ sucrose solutions, and centrifuged at $85,000 \times g$ for $2 \mathrm{~h}\left(4^{\circ} \mathrm{C}\right)$, using an SW-28 swinging bucket rotor. The synaptosomal layer at the $1.0 / 1.2 \mathrm{M}$ sucrose interface was collected, aliquoted, and stored at $-80^{\circ} \mathrm{C}$.

Protein concentration was determined using the Bradford assay. Protein extracts were separated on either 4-12\% Bis-Tris gels (for analysis of synaptophysin and MAP2) or 3-8\% Tris-acetate gels (for analysis of Piccolo and PSD95) (Invitrogen NuPAGE Novex gels). In each well, either $50 \mu \mathrm{g}$ of whole-brain protein or $4 \mu \mathrm{g}$ of synaptosomal protein was loaded. Subsequently, proteins were transferred to a nitrocellulose membrane (Hybond ECL; Amersham Biosciences, Piscataway, NJ). The membranes were washed in TBS, blocked in TBS containing 5\% milk, and probed with rabbit anti-MAP2 (1:1500), rabbit anti-Piccolo (1:1000), mouse anti-PSD95 (1:2000), and mouse anti-synaptophysin (1:4000). Detection of bound primary antibodies was performed with fluorescently conjugated goat anti-rabbit (Alexa Fluor 680, 1:30,000; Invitrogen) or goat anti-mouse secondary antibodies (IRDye 800, 1:5000; Rockland Immunochemicals, Gilbertsville, PA). Immunofluorescence was then detected and analyzed using the Odyssey Imaging System (LI-COR Biosciences, Lincoln, NE). Samples from all animals were run together on each gel, and gels were replicated three to five times. Control wells with half and double the protein concentration were loaded onto each gel to ensure that the intensity of all of the protein bands fell within a linear range of detection.

Statistics. Comparison of group means from immunohistochemical results was performed with ANOVA ( $n=6$ animals per group). A post hoc correction for multiple comparisons was made using Fisher's least significant difference method. Quantitative Western blots were analyzed using a two-tailed $t$ test ( $n=3$ animals per group). The precision of intensity of Western blot bands was $\pm 3 \%$ (SEM).

\section{Results}

Torpor-related loss of synaptic protein clustering is similar in four brain regions

We investigated immunohistochemical changes of several synaptic proteins in four regions of the squirrel brain: cortical layer 4 in the somatosensory cortex, the somatosensory ventral posterior thalamic relay nucleus, the reticular nucleus of the thalamus, and hippocampus CA3 stratum oriens. Changes in synaptic protein immunoreactivity in torpor have been observed previously in the CA3 region of the hippocampus (Arendt et al., 2003). We investigated whether these changes are part of a more global phenomenon.

We analyzed dynamics of torpor-related changes in four proteins: MAP2, Piccolo, PSD95, and synaptophysin. MAP2 is a structural protein that inter-links dendritic microtubules (Garner et al., 1988) and provides information about the state of dendritic arbors. Piccolo and synaptophysin provide information about the condition of presynaptic structures: Piccolo is a 
core cytomatrix protein found in the presynaptic active zone (Cases-Langhoff et al., 1996), and synaptophysin is a presynaptic vesicle glycoprotein (Wiedenmann and Franke, 1985). PSD95 is a postsynaptic density protein important for the trafficking and stabilization of glutamate receptors (Kornau et al., 1995) and provides information about the condition of the postsynaptic structure.

To compare torpor-related changes in synaptic protein clustering among the aforementioned brain regions, we used two groups of animals. The first group was killed $6 \mathrm{~d}$ into a torpor bout at $5^{\circ} \mathrm{C}$, and the second group was killed $12 \mathrm{~h}$ after the initiation of arousal to euthermic temperatures. Representative immunohistochemical images from the four brain regions are shown in Figure $1 A$.

All four regions exhibited torporrelated reductions in area covered by synaptic protein clusters, as shown in Figure 2 ( $p<0.05$ for all comparisons between late euthermia and late torpor, except for synaptophysin in the ventral posterior thalamus and Piccolo in the cortex and ventral posterior thalamus). The amount of decrease was similar among the four regions, as determined by both extent of decrease ( $p>0.05$ for all comparisons) (Fig. 2) and percentage decline ( $p>0.05$ for all comparisons) (Fig. 3) of puncta coverage. These findings suggest that torpor-related structural changes are taking place in a similar manner generally across the squirrel brain.

We analyzed whether the torporrelated decrease in area covered by synaptic protein clusters is attributable primarily to a change in the number of puncta or in the size of these puncta. For the three punctate proteins analyzed (Piccolo, PSD95, and synaptophysin), both the number and size of puncta decreased in torpor in all of the brain regions. The decline in puncta number reached statistical significance for synaptophysin (cortex, $p=0.002$; ventral posterior thalamus, $p=0.016$; reticular nucleus of the thalamus, $p=0.004$; hippocampus, $p=$ 0.011 ) and PSD95 (cortex, $p=0.015$; ventral posterior thalamus, $p=0.039$ ). Thus, the torpor-related loss of area covered by synaptic protein clusters appears to be attributable to a loss of protein from individual puncta.

\section{Torpor-related changes in synaptic protein clustering track body temperature}

We investigated the time course of torpor-related changes in synaptic protein clustering by examining immunohistochemical images from animals at five different time points in the torpor cycle at $5^{\circ} \mathrm{C}: 1,3$, and $6 \mathrm{~d}$ after torpor entrance and 2 and $12 \mathrm{~h}$ after arousal induction. These time points correspond to the start and end of both torpor and interbout euthermia: the torpor cycle of a golden-mantled ground squirrel hibernating at $5^{\circ} \mathrm{C}$ consists of a $1 \mathrm{~d}$ entry phase, a 5-7 d interval of deep torpor at a body temperature of $\sim 7^{\circ} \mathrm{C}$, a $2 \mathrm{~h}$ spontaneous return to euthermia, and an
8-12 h interbout euthermic interval, followed by torpor reentry. A sample body temperature graph from a hibernating squirrel is provided in Figure $2 E$.

The time course for torpor-related changes in the area covered by synaptic protein clusters is shown in Figure 2. The majority of the decrease took place by day 1, a finding that was, again, consistent among the brain regions investigated. This decrease by day 1 was statistically significant (at $\alpha=0.05$ ) for MAP2 in all regions, for Piccolo in the reticular nucleus of the thalamus, for PSD95 in the cortex, reticular nucleus of the thalamus, and hippocampus, and for synaptophysin in the hippocampus. After entrance into torpor, no statistically significant change occurred over the course of several days in deep torpor. Overall, synaptic protein immunoreactivity in torpor was $37-55 \%$ lower than that in euthermia; synaptophysin demonstrated the greatest percentage decrease from euthermia, whereas Piccolo demonstrated the least. We conclude that there is significant loss of synaptic protein clustering as the brain cools during entry into torpor rather than gradually over several days at low temperature.

The majority of the recovery of synaptic protein clustering took place within the short $2 \mathrm{~h}$ arousal window from torpor, doing so globally across brain regions. This increase reached statistical significance for MAP2 in all regions and for PSD95 in the 


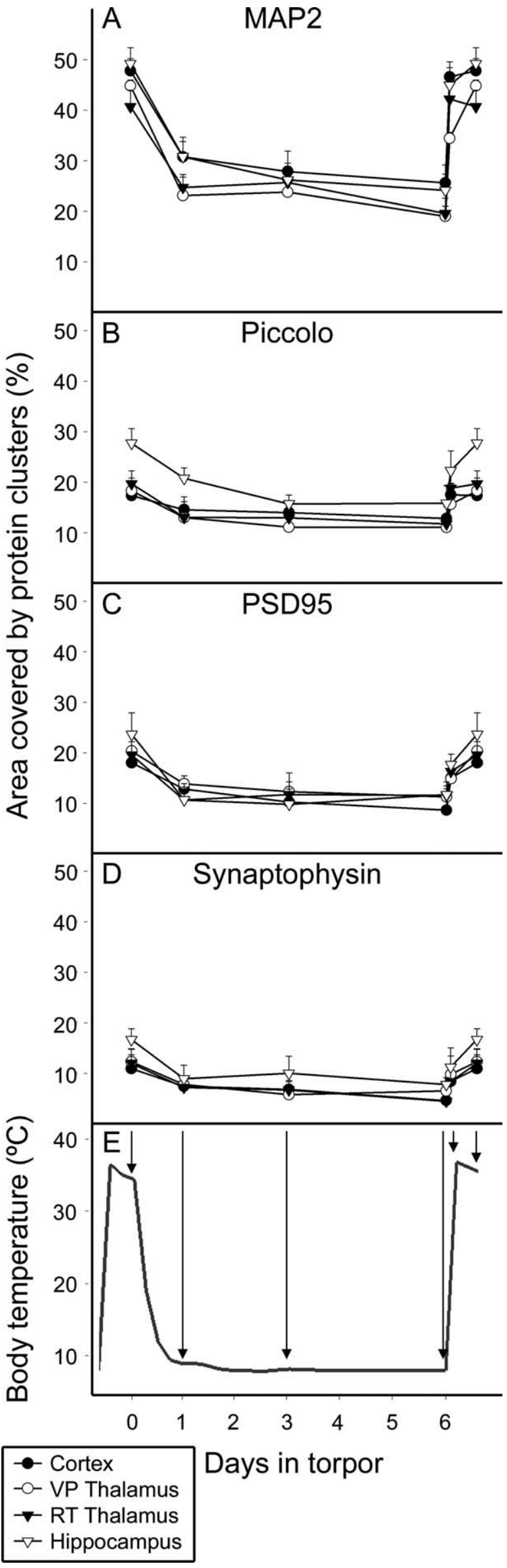

reticular nucleus of the thalamus. The $2 \mathrm{~h}$ recovery ranged from $52 \%$ of euthermic values for synaptophysin to $86 \%$ of euthermic values for MAP2. Because torpor values were much lower than euthermic values, achieving the majority of this recovery in $2 \mathrm{~h}$ required a high rate of increase. The amount of MAP2, for example, nearly doubled during the $2 \mathrm{~h}$ arousal window. We conclude that the majority of synaptic protein recovery from torpor takes place as the body is warming during arousal.

\section{Protein clustering decreases linearly with minimum torpor body temperature}

The ambient temperature during torpor determines minimum body temperature (Strumwasser, 1959). Minimum body temperature, in turn, determines many physiological and behavioral parameters, including torpor bout length (Buck and Barnes, 2000) and neural activity (Krilowicz et al., 1988). The ambient temperature during hibernation also influences the extent of neural structural plasticity during torpor (von der Ohe et al., 2006). In this experiment, we assessed whether plasticity of synaptic protein clustering in hibernation is also temperature dependent.

We analyzed immunohistochemical images from animals $3 \mathrm{~d}$ into a torpor bout at 5 and $15^{\circ} \mathrm{C}$ (Fig. $3 \mathrm{~A}$ ) to assess whether there are temperature-dependent differences in area covered by protein clusters. In all four brain regions, there was a temperaturedependent trend in protein clustering: the area covered by synaptic protein hotspots from animals hibernating at $5^{\circ} \mathrm{C}$ was consistently lower than that from animals hibernating at $15^{\circ} \mathrm{C}$ (Fig. 3). Analysis of the percentage decrease of immunoreactivity from euthermia as a function of torpor body temperature suggests a linear relationship ( $p<0.05$ for linear regression for each of the proteins and brain regions investigated), shown in Figure 3 . Analysis of the slopes of torpor-related decrease across proteins and brain regions revealed that there were no differences among the proteins ( $p=0.518$ ), as well as among the brain regions ( $p=$ $0.495)$. We conclude that synaptic clustering decreases linearly with temperature at a rate that is similar across all proteins and brain regions investigated.

To assess whether the area covered by synaptic protein clusters recovers to fixed values, regardless of the extent of puncta loss in the previous torpor bout, we compared immunofluorescence in the 5 and $15^{\circ} \mathrm{C}$ groups $12 \mathrm{~h}$ after arousal. There were no significant differences between these two groups of animals across all of the synaptic proteins and across all of the brain regions analyzed (at $\alpha=0.05$ ). This finding suggests the existence of a mechanism that determines final synaptic protein density during recovery from torpor.

Figure 2. Time course of changes in synaptic protein clustering. $A-D$, Torpor-related dynamics of the area covered by protein clusters are shown for MAP2 (A), Piccolo (B), PSD95 (C), and synaptophysin $(\boldsymbol{D})$ (mean $+\mathrm{SEM} ; n=6$ ). $\boldsymbol{E}$, Time points investigated correspond to specific times in the torpor bout cycle. Means are from images from the four regions investigated: layer 4 somatosensory cortex, ventral posterior (VP) thalamus, reticular (RT) nucleus of the thalamus, and hippocampus CA3 (see key). Synaptic protein clustering decreases significantly during the $1 \mathrm{~d}$ entry phase ( $p<0.05$ for MAP2 in all regions, for Piccolo in the reticular nucleus of the thalamus, for PSD95 in the cortex, reticular nucleus of the thalamus, and hippocampus, and for synaptophysin in the hippocampus). After that time, there is no decrease in puncta coverage in torpor for any of these proteins in any of these regions. More than $50 \%$ of the recovery takes place in the $2 \mathrm{~h}$ arousal window, with significant increases $(\alpha=0.05)$ in MAP2 in all regions and for PSD95 in the reticular nucleus of the thalamus. 


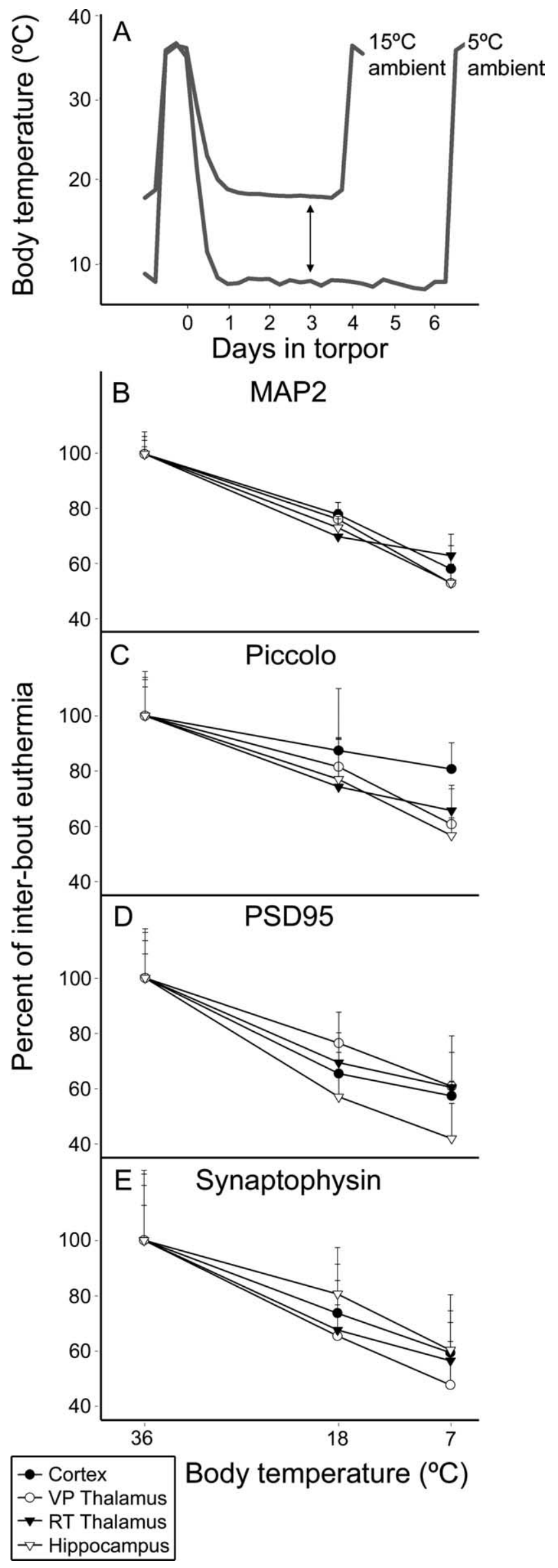

Torpor-related loss of colabeled presynaptic and postsynaptic puncta

We investigated whether the loss of synaptic protein clusters in torpor affects synapse number, as defined by the colocalization of presynaptic and postsynaptic protein puncta. We analyzed two groups of animals: the first group was killed $6 \mathrm{~d}$ into a torpor bout at $5^{\circ} \mathrm{C}$ and the second group was killed $12 \mathrm{~h}$ after initiation of arousal to euthermic temperatures. Double labeling of slices from the four brain regions described above were performed with the presynaptic protein Piccolo and the postsynaptic protein PSD95. Torpid animals exhibited a $50-65 \%$ decline in colabeled puncta relative to euthermic animals ( $p<0.001$ for all regions), as shown in Figure 4.

We tested whether this large decline of colabeled puncta could be accounted for merely by the torpor-related decrease in number of Piccolo and PSD95 puncta or whether colabeled puncta were targeted. The same analysis was repeated with the PSD95 image rotated by $90^{\circ}$, while the Piccolo image remained in its original orientation. This analysis ensured that presynaptic and postsynaptic puncta were not functionally related, enabling analysis of the torpor-related percentage decrease in randomly overlapping colabeled puncta. There was a $26 \%$ loss of randomly colabeled puncta, demonstrating that torpor results in a significant loss of synapses.

Total levels of synaptic protein immunofluorescence do not change with torpor

We analyzed whether the loss of area covered by synaptic protein clusters is attributable to an overall loss of immunofluorescence in slices from torpid animals. The measurement of area covered by immunofluorescent clusters was made after subtracting background levels of immunofluorescence, which left discrete hotspots of fluorescence that are thought to correspond to synapses. When we analyzed the intensity of immunofluorescence without subtracting background levels of immunofluorescence, we found that there were no differences between euthermic and hibernating animals (Table 1), indicating that overall levels of fluorescence, and thus the overall amount of these synaptic proteins, do not change between these groups. Together with the loss of area covered by discrete protein clusters during torpor, these data indicate that Piccolo, PSD95, and synaptophysin shift from a punctate, or synaptic, organization during euthermia to a more diffuse nonsynaptic pool of protein during torpor. The distribution of MAP2, a nonsynaptic protein, also changes markedly during torpor, as can be seen in Figure 1. MAP2 shifts from an organized and filamentous pattern in the euthermic animal (Fig. $1 B$ ) to a punctate and less organized pattern in the torpid animal (Fig. $1 C)$. The change in distribution of these proteins likely results in the loss of high-intensity immunofluorescence during hibernation.

\footnotetext{
Figure 3. Temperature dependency of synaptic protein dynamics. $\boldsymbol{A}$, Body temperature graphs from animals hibernating at 5 and $15^{\circ} \mathrm{C}$, with arrows indicating the experimental groups for comparison of the effects of temperature on synaptic protein clustering. The relationship of the area covered by synaptic protein clusters with torpor body temperature is shown for MAP2 $(\boldsymbol{B})$, Piccolo $(\boldsymbol{C})$, PSD95 (D), and synaptophysin $(\boldsymbol{E})$ (mean + SEM; $n=6$ ). Means are from images from the four regions investigated: layer 4 somatosensory cortex, ventral posterior (VP) thalamus, reticular (RT) nucleus of the thalamus, and hippocampus CA3 (see key). The slopes of torpor-related decrease were similar among the brain regions ( $p=0.495)$, as well as among the proteins investigated ( $p=0.518$ ).
} 

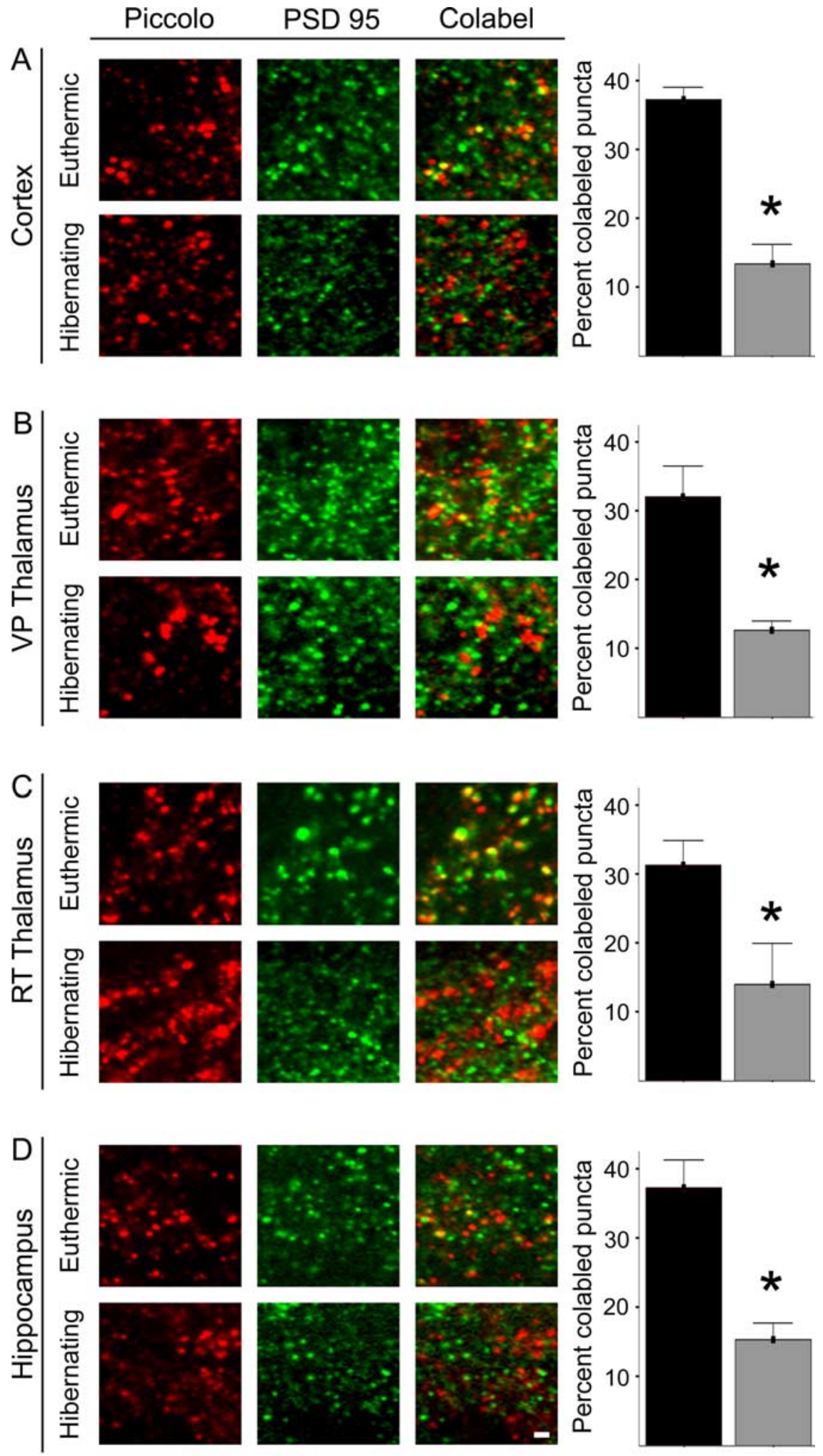

Euthermic DHibernating

Figure 4. Colabeling of presynaptic and postsynaptic proteins. Double labeling was performed with Piccolo and PSD95. Images of stains from each of these proteins are shown individually and colabeled for both euthermic ( $12 \mathrm{~h}$ after arousal induction) and torpid ( $6 \mathrm{~d}$ after torpor entrance) animals hibernating at $5^{\circ} \mathrm{C}$. The percentage of puncta that were colabeled are shown on the right (mean $+\mathrm{SEM} ; n=6)$. Sets of images and graphs are from the four regions investigated: layer 4 somatosensory cortex, ventral posterior (VP) thalamus, reticular (RT) nucleus of the thalamus, and hippocampus CA3. Scale bar, $1 \mu \mathrm{m} .{ }^{*} p<0.005$, statistically significant decreases.
Torpor-related loss of synaptic protein clusters is not attributable to protein loss

To confirm that the loss of synaptic protein clusters during entrance into torpor is not attributable to net protein loss during entrance into torpor, we performed quantitative Western blots of MAP2, Piccolo, PSD95, and synaptophysin from animals $1 \mathrm{~d}$ into a torpor bout at $5^{\circ} \mathrm{C}$ and $12 \mathrm{~h}$ after arousal. Fractions containing whole-brain protein and synaptosomal protein were analyzed independently. Breakdown products of MAP2 were also analyzed to determine whether proteolytic processes are active during entrance into torpor.

No significant differences in protein concentrations of the main band of any of these proteins were found in both wholebrain and synaptosomal fractions, shown in Figure 5 ( $p>0.05$ for all comparisons). This indicates that the loss of synaptic protein clustering demonstrated by immunohistochemistry is not attributable to net protein loss.

To determine whether proteolysis does occur during entrance into torpor, we analyzed three MAP2 breakdown products at 170,180 , and $200 \mathrm{kDa}$. An increase in these breakdown products would indicate that proteolytic processes are active during entrance into torpor, although this would be balanced by protein synthesis because main band intensities were shown to remain constant. Each of the three MAP2 breakdown products demonstrated an increase in density of $10-20 \%$ during entrance into torpor in the synaptosomal fraction ( $p<0.05$ for all three breakdown products). In the whole-brain fraction, there was a $5-10 \%$ trend in increased breakdown products, but this did not reach statistical significance. To account for slight differences in main band density, we analyzed the ratio of breakdown product to main band density and found a significant $5-15 \%$ torpor-related increase in this ratio in the synaptosomal fractions of all three breakdown products $(p<0.05$ for each breakdown product). The torporrelated increase in this ratio in wholebrain protein fractions did not reach statistical significance. We conclude that protein breakdown continues to take place during some portion of the entrance phase into torpor but that it is balanced by protein synthesis, resulting in the absence of net protein loss.

\section{Discussion}

Our findings enhance our understanding of the hibernator as a model of adult neural plasticity. We show that changes in area 
covered by synaptic protein clusters are of similar magnitude in different brain regions and that these changes occur similarly in presynaptic and postsynaptic structures, suggesting that that this phenomenon is taking place generally across the brain. We show that the changes take place in a temperature-dependent manner, with greater loss of synaptic protein clusters at lower hibernation temperatures. We demonstrate a loss of synaptic protein coclusters during torpor, implying not only a loss of synapses but a requirement for a massive restructuring of neural connections with each arousal from torpor. Last, we show that the loss of synaptic protein clusters that occurs during entry into torpor is not attributable to protein degradation. Our results suggest that synaptic proteins dissociate from the synapse to the axon, soma, or dendrite during entrance into torpor, remain in that state for the duration of the torpor bout, and then reassemble rapidly during arousal from torpor.

Our finding that the loss of synaptic clustering during torpor is a global phenomenon suggests that a common trigger is at work across the entire brain. This common trigger may be temperature. Our data indicate that synaptic protein clustering closely tracks body temperature, which is consistent with the time course demonstrated by Arendt et al. (2003) in the European ground squirrel. We also show that the extent of loss of puncta coverage is dependent on the minimum body temperature during torpor. Together, these findings strongly suggest a temperature-driven model. Temperature may cause dissociation of synaptic proteins either directly through some unknown mechanism or indirectly through the temperaturedependent dendritic retraction demonstrated by von der Ohe et al. (2006).

Support for the presence of an indirect mechanism comes from a study in acute hippocampal slice preparations, in which cooling resulted in the appearance of floating postsynaptic densities in the dendrite (Kirov et al., 2004). Thus, microstructural changes as a result of cooling may cause displacement of synaptic proteins. One area of application for this research is hypothermia injury in humans. Patients recovering from hypothermia have been shown to suffer cognitive impairments (Walpoth et al., 1997). Our results suggest that these impairments may be the result of temperature-dependent loss of neural connectivity, highlighting the need for research efforts in this area.

The data presented in this paper show that the extensive loss of synaptic protein clustering during torpor is not attributable to loss of these proteins. Rather, it appears to be attributable to a mechanism of dissociation of proteins from their synapses into the axon, dendrite, or cell body. This conclusion is based on findings from both immunohistochemistry and Western blot analysis. First, the overall immunofluorescence intensity does not differ between euthermic and torpid animals, despite a loss of area covered by protein clusters. This indicates that the proteins are still present during torpor but are no longer organized in clusters. These changes can be visualized in the immunohistochemistry images, in which torpid animals demonstrate an increase in background staining for the punctate proteins and a loss of filamentous organization for MAP2. Second,
Table 1. Fluorescence intensity of immunohistochemistry images

\begin{tabular}{lll}
\hline & Euthermic & Hibernating \\
\hline MAP2 & $1577.06 \pm 203.53$ & $1518.22 \pm 169.66$ \\
Piccolo & $1233.39 \pm 131.87$ & $1181.89 \pm 137.66$ \\
PSD95 & $1728.56 \pm 200.90$ & $1870.78 \pm 191.83$ \\
Synaptophysin & $1072.22 \pm 95.87$ & $1151.17 \pm 82.56$ \\
\hline
\end{tabular}

Fluorescence intensities of immunohistochemistry images without subtraction of background levels of fluorescence. Mean \pm SEM are from groups of six euthermic animals ( $12 \mathrm{hr}$ after arousal) and six hibernating animals ( $6 \mathrm{~d}$ after torpor entry). Proteins investigated are MAP2, Piccolo, PSD95, and synaptophysin. Fluorescence intensity was averaged from three $625 \mu \mathrm{m}^{2}$ squares per image across the six animals per group.
Synaptosomes

Hibernating
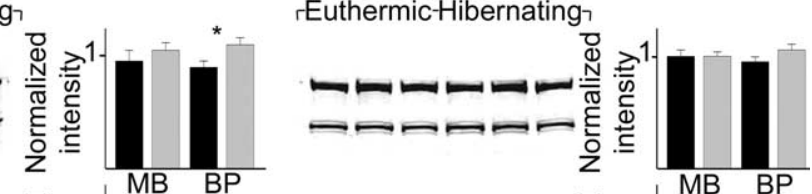
rEuthermic-Hibernating
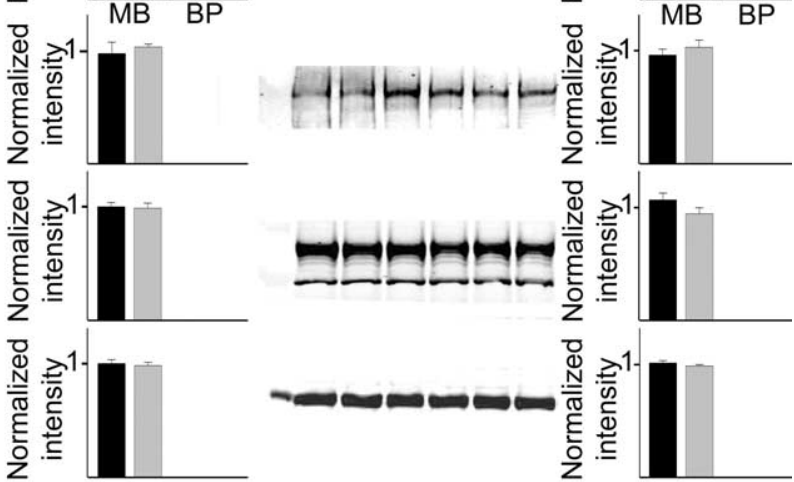

西

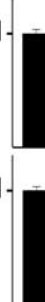

i
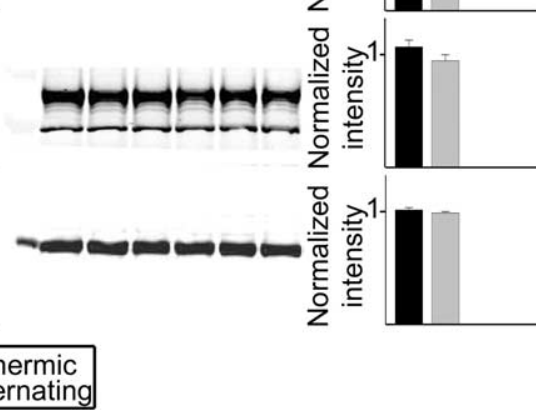

Euthermic

Figure 5. Western blots of proteins. Protein bands from both synaptosomal and whole-brain fractions are shown. Blots are of the four proteins: MAP2, Piccolo, PSD95, and synaptophysin. The first three bands are from euthermic animals ( $12 \mathrm{~h}$ after arousal), right of every set of blots are graphs of normalized intensity values of bands from euthermic and hibernating animals across three gels (mean + SEM). For MAP2, data for the main band (MB) as well as the breakdown product (BP) at $180 \mathrm{kDa}$ are shown. ${ }^{*} p<$ 0.05 , statistically significant difference between euthermic and hibernating animals.

Western blots of all four proteins demonstrate that their concentrations do not change with entrance into torpor. Although data from the analysis of MAP2 breakdown products indicate that proteolytic processes continue to be active during some portion of the entrance phase, protein breakdown appears to be balanced by protein synthesis because the concentration of main protein remains constant. Thus, it appears that the proteins are still present during torpor but that they have assumed a different, less clustered, organization.

To our knowledge, a protein-dissociation-based model for large-scale neural retraction and synaptic loss has not been demonstrated previously. Other models of synaptic plasticity generally involve the regulated disassembly of large protein complexes via proteosomal or lysosomal degradation (DiAntonio et al., 2001; Burbea et al., 2002; Watts et al., 2003). Select synaptic proteins do exist in mobile pools in the neuron and are available during synapse assembly, although it is unknown whether these proteins are synthesized de novo or remobilized from other synapses. In the axon, for example, rapid recruitment of preassembled synaptic components are brought to synapses via mobile transport packets containing some synaptic vesicle proteins and active zone components, including Piccolo, Bassoon, and $\mathrm{N}$-cadherin (Ahmari et al., 2000; Zhai et al., 2001). In the dendrite, both PSD95 clusters and AMPA receptors can be recruited from a diffuse cytoplasmic pool (Bresler et al., 2001; Marrs et al., 
2001; Borgdorff and Choquet, 2002), and NMDA receptor subunits are available in discrete dendritic transport packets (Washbourne et al., 2002). Similar nonsynaptic pools also contribute to dynamic exchange rates of synaptic proteins at mature synapses (Tsuriel et al., 2006). However, it has never before been demonstrated that synaptic proteins can rapidly and repeatedly disassemble and reassemble to cause large-scale microstructural and synaptic changes.

These dissociated synaptic proteins may serve as a reservoir for rebuilding synapses during arousal from torpor. This finding could explain the speed of the recovery process, the majority of which occurs in just $2 \mathrm{~h}$ during the return to euthermia. In addition, the fact that proteolysis and protein synthesis are not the dominant mechanisms for neural plasticity in hibernation is consistent with what is known about the energetic requirements of hibernation. Hibernation is a state of extremely low energy availability (Humphries et al., 2003); therefore, it is not surprising that the hibernator accomplishes repeated and large-scale neural structural changes without the energetically costly processes of proteolysis and protein synthesis. A mechanism of neural plasticity based on protein dissociation rather than protein breakdown could explain the hibernator's capacity for rapid and energetically efficient microstructural changes.

The finding that the loss of synaptic connectivity in torpor is not attributable to protein loss suggests that this process is not the result of degenerative or cellular stress pathways. Although neurons undergoing programmed cell death exhibit dendritic retraction similar to neurons in the hibernator (Nuydens et al., 1997; Pena and Pilar, 2000), cell death processes are associated with the activation of multiple protein breakdown pathways (Chan and Mattson, 1999). Some of these pathways lead to the breakdown of structural proteins, likely causing the cytoskeletal derangement and functional loss seen in some degenerative conditions (for review, see Wang 2000). The current study has uncovered a mechanism that distinguishes the neural changes in the hibernator from those seen in neuropathologies in which protein breakdown and cell death appear to be irreversible processes. Understanding the mechanisms for reversible protein dissociation may provide insights into potential therapeutic interventions in neurodegenerative disorders.

Our results show that neural plasticity in hibernation results in a dramatic loss of apparent synapses, likely resulting in largescale restructuring of neural connections with each arousal from torpor. These findings may shed light on previous studies of longterm memory in hibernation. Ground squirrels that hibernate exhibit reduced performance in hippocampal-dependent memory tasks relative to those that do not hibernate (Millesi et al., 2001). Ground squirrels also demonstrate a diminished ability after hibernating to recognize previously familiar squirrels, although they retain the ability to recognize littermates (Mateo and Johnston, 2000). It is possible that the loss of memory associated with hibernation is related to the large-scale neural changes. This would suggest that the rapid recovery of synaptic clusters to a fixed set of values does not necessarily mean that all types of information in the synapses are retained. The temperaturedependent loss of synapses shown here opens the door to investigating where and how memories are stored, which types of memories are most sensitive to structural loss, and whether synaptic connections can regrow to their previous configuration.

In conclusion, we have shown that the dramatic and widespread neural plasticity demonstrated in the hibernator is a result of dissociation of synaptic proteins from synapses and that these proteins may serve as a reservoir for rapid and energetically effi- cient rebuilding of those synapses. We also demonstrated that this model of neural plasticity can be manipulated by altering ambient temperature, resulting in a predictable extent of synaptic loss that is large in both rate and magnitude. Our results have improved our understanding of the adult neural plasticity in the hibernator and have advanced the potential use of this model system for learning about processes including hypothermia, learning and memory, and neurodegeneration.

\section{References}

Ahmari SE, Buchanan J, Smith SJ (2000) Assembly of presynaptic active zones from cytoplasmic transport packets. Nat Neurosci 3:445-451.

Arendt T, Stieler J, Strijkstra AM, Hut RA, Rudiger J, Van der Zee EA, Harkany T, Holzer M, Hartig W (2003) Reversible paired helical filamentlike phosphorylation of tau is an adaptive process associated with neuronal plasticity in hibernating animals. J Neurosci 23:6972-6981.

Barnes BM (1989) Freeze avoidance in a mammal: body temperatures below 0 degree $C$ in an Arctic hibernator. Science 244:1593-1595.

Borgdorff AJ, Choquet D (2002) Regulation of AMPA receptor lateral movements. Nature 417:649-653.

Bresler T, Ramati Y, Zamorano PL, Zhai R, Garner CC, Ziv NE (2001) The dynamics of SAP90/PSD-95 recruitment to new synaptic junctions. Mol Cell Neurosci 18:149-167.

Buck CL, Barnes BM (2000) Effects of ambient temperature on metabolic rate, respiratory quotient, and torpor in an arctic hibernator. Am J Physiol Regul Integr Comp Physiol 279:R255-R262.

Burbea M, Dreier L, Dittman JS, Grunwald ME, Kaplan JM (2002) Ubiquitin and AP180 regulate the abundance of GLR-1 glutamate receptors at postsynaptic elements in C. elegans. Neuron 35:107-120.

Cases-Langhoff C, Voss B, Garner AM, Appeltauer U, Takei K, Kindler S, Veh RW, De Camilli P, Gundelfinger ED, Garner CC (1996) Piccolo, a novel $420 \mathrm{kDa}$ protein associated with the presynaptic cytomatrix. Eur J Cell Biol 69:214-223.

Chan SL, Mattson MP (1999) Caspase and calpain substrates: roles in synaptic plasticity and cell death. J Neurosci Res 58:167-190.

DiAntonio A, Haghighi AP, Portman SL, Lee JD, Amaranto AM, Goodman CS (2001) Ubiquitination-dependent mechanisms regulate synaptic growth and function. Nature 412:449-452.

Garner CC, Tucker RP, Matus A (1988) Selective localization of messenger RNA for cytoskeletal protein MAP2 in dendrites. Nature 336:674-677.

Humphries MM, Kramer DL, Thomas DW (2003) The role of energy availability in Mammalian hibernation: an experimental test in free-ranging eastern chipmunks. Physiol Biochem Zool 76:180-186.

Kirov SA, Petrak LJ, Fiala JC, Harris KM (2004) Dendritic spines disappear with chilling but proliferate excessively upon rewarming of mature hippocampus. Neuroscience 127:69-80.

Kornau HC, Schenker LT, Kennedy MB, Seeburg PH (1995) Domain interaction between NMDA receptor subunits and the postsynaptic density protein PSD-95. Science 269:1737-1740.

Krilowicz BL, Glotzbach SF, Heller HC (1988) Neuronal activity during sleep and complete bouts of hibernation. Am J Physiol 255:R1008-R1019.

Marrs GS, Green SH, Dailey ME (2001) Rapid formation and remodeling of postsynaptic densities in developing dendrites. Nat Neurosci 4:1006-1013.

Mateo JM, Johnston RE (2000) Retention of social recognition after hibernation in Belding's ground squirrels. Anim Behav 59:491-499.

Millesi E, Prossinger H, Dittami JP, Fieder M (2001) Hibernation effects on memory in European ground squirrels (Spermophilus citellus). J Biol Rhythms 16:264-271.

Nuydens R, Dispersyn G, de Jong M, van den Kieboom G, Borgers M, Geerts H (1997) Aberrant tau phosphorylation and neurite retraction during NGF deprivation in PC12 cells. Biochem Biophys Res Commun 240:687-691.

Pena C, Pilar G (2000) Early morphologic alterations in trophically deprived neuronal death in vitro occur without alterations in cytoplasmic $\mathrm{Ca}^{2+}$. J Comp Neurol 424:377-396.

Popov VI, Bocharova LS, Bragin AG (1992) Repeated changes of dendritic morphology in the hippocampus of ground squirrels in the course of hibernation. Neuroscience 48:45-51.

Shtark MB (1970) The brain of hibernating animals. Novosibirsk, Siberia: Nauka. 
Strumwasser F (1959) Regulatory mechanisms, brain activity and behavior during deep hibernation in the squirrel, Citellus beecheyi. Am J Physiol 196:23-30.

Tsuriel S, Geva R, Zamorano P, Dresbach T, Boeckers T, Gundelfinger ED, Garner CC, Ziv NE (2006) Local sharing as a predominant determinant of synaptic matrix molecular dynamics. PLoS Biol 4:e271.

von der Ohe CG, Darian-Smith C, Garner CC, Heller HC (2006) Ubiquitous and temperature-dependent neural plasticity in hibernators. J Neurosci 26:10590-10598.

Walker JM, Glotzbach SF, Berger RJ, Heller HC (1977) Sleep and hibernation in ground squirrels (Citellus spp): electrophysiological observations. Am J Physiol 233:R213-R221.

Walpoth BH, Walpoth-Aslan BN, Mattle HP, Radanov BP, Schroth G, Schaeffler L, Fischer AP, von Segesser L, Althaus U (1997) Outcome of survivors of accidental deep hypothermia and circulatory arrest treated with extracorporeal blood warming. N Engl J Med 337:1500-1505.
Wang KK (2000) Calpain and caspase: can you tell the difference? Trends Neurosci 23:20-26.

Wang LC (1973) Radiotelemetric study of hibernation under natural and laboratory conditions. Am J Physiol 224:673-677.

Washbourne P, Bennett JE, McAllister AK (2002) Rapid recruitment of NMDA receptor transport packets to nascent synapses. Nat Neurosci 5:751-759.

Watts RJ, Hoopfer ED, Luo L (2003) Axon pruning during Drosophila metamorphosis: evidence for local degeneration and requirement of the ubiquitin-proteasome system. Neuron 38:871-885.

Wiedenmann B, Franke WW (1985) Identification and localization of synaptophysin, an integral membrane glycoprotein of Mr 38,000 characteristic of presynaptic vesicles. Cell 41:1017-1028.

Zhai RG, Vardinon-Friedman H, Cases-Langhoff C, Becker B, Gundelfinger ED, Ziv NE, Garner CC (2001) Assembling the presynaptic active zone: a characterization of an active one precursor vesicle. Neuron 29:131-143. 